\title{
O trabalho e a tecnologia assistiva na perspectiva de pessoas com deficiência física*
}

\section{Work and assistive technology on perspective of people with physical disabilities}

\author{
Daniel Marinho Cezar da Cruz ${ }^{1}$, Daniela da Silva Rodrigues ${ }^{2}$, Alessandra Mary \\ Matsushima $^{3}$, Patrícia dos Santos ${ }^{4}$, Mirela de Oliveira Figueiredo ${ }^{5}$
}

http://dx.doi.org/10.11606/issn.2238-6149.v26i3p382-389

\begin{abstract}
Cruz DMC, Rodrigues DS, Matsushima AM, Santos P, Figueiredo MO. O trabalho e a tecnologia assistiva na perspectiva de pessoas com deficiência física. Rev Ter Ocup Univ São Paulo. 2015 set.dez.;26(3):382-9.
\end{abstract}

RESUMO: Nos dias atuais, a empregabilidade das pessoas com deficiência é um debate crescente e as dificuldades no trabalho precisam ser compreendidas a partir daqueles que vivenciam esse processo. Objetiva-se identificar os aspectos que facilitam e/ou dificultam a inclusão de pessoas com deficiência em atividades laborais e entender a contribuição da tecnologia assistiva no desempenho do papel ocupacional de trabalho. Trata-se de uma pesquisa qualitativa. Participaram 30 sujeitos, com idade entre 18 e 60 anos. Foi desenvolvida entrevista semiestruturada e a análise dos dados foi feita a partir da análise de conteúdo temática. O trabalho apresentou-se como importante meio de realização pessoal e profissional na vida dos entrevistados, além de forma de reconhecimento pela sociedade. A tecnologia assistiva apareceu como um facilitador tanto no ambiente de trabalho quanto em relação à acessibilidade para se chegar a ele. A escolaridade e o preconceito foram pontuados como as principais barreiras ao processo de inclusão. Destaca-se a importância de ações afirmativas e de incentivo ao debate de políticas públicas pelo acesso aos direitos das pessoas com deficiência, garantidos na Lei brasileira.

DESCRITORES: Pessoas com deficiência; Tecnologia/métodos; Equipamentos de autoajuda; Terapia ocupacional/métodos; Trabalho.
Cruz DMC, Rodrigues DS, Matsushima AM, Santos P, Figueiredo MO. Work and assistive technology on perspective of people with physical disabilities. Rev Ter Ocup Univ São Paulo. 2015 Sept.Dec.;26(3):382-9.

\begin{abstract}
The employability of people with disabilities is an important debate nowadays, and the difficulties, facilitators and barriers at work are issues that need to be understood from those who have gone through this process. Aim to identify through the perspective of people with physical disability aspects that facilitate and/or hinder their inclusion in work activities and understand the contribution of assistive technology in the performance of the role of work for these people. There were 30 participants aged between 18 and 60 years with physical disability, who were interviewed by the researchers. The data analysis for the interviews was performed using the content analysis. Results: The work is presented as an important means of personal and professional fulfillment in the lives of respondents, and a way of inclusion in society. Assistive technology appeared as facilitator both on relevant issues in the workplace and in relation to accessibility, such as adapted transport and mobility. Education and prejudice were identified as the main barriers to the inclusion process at work. It highlights the importance of affirmative action and to encourage the debate of public policies for access to rights of people with disabilities, guaranteed by Brazilian law.
\end{abstract}

KEYWORDS: Disabled persons; Self-help devides; Technology/ methods; Occupational therapy/methods; Work.

\footnotetext{
*Este trabalho é parte integrante de pesquisa de iniciação científica 2013-2014, do Programa de Bolsas de Iniciação Científica- PIBIC da Universidade Federal de São Carlos. Financiamento - CNPq.

1. Universidade Federal de São Carlos, Departamento de Terapia Ocupacional, Programa de Pós-Graduação em Terapia Ocupacional, Laboratório de Análise Funcional e Ajudas Técnicas - LAFATec.

2. Universidade de Brasília - UnB, Faculdade Ceilândia, Laboratório de Análise Funcional e Ajudas Técnicas - LAFATec.

3. Universidade Federal de São Carlos, Laboratório de Análise Funcional e Ajudas Técnicas- LAFATec. Bolsista PIBIC- CNPq, UFSCar.

4. Universidade Federal de São Carlos - UFSCar, Curso de Terapia Ocupacional, aluna Bolsista PIBIC - CNPq.

5. Universidade Federal de São Carlos.

Endereço para correspondência: Daniel Marinho Cezar da Cruz. Rua Cristiano Viana, 450 apto. 61. Cerqueira Cesar - São Paulo, SP, Brasil. CEP: 05411-000. E-mail: cruzdmc@gmail.com
} 


\section{INTRODUÇÃO}

$\mathbf{O}$ trabalho apresenta diversos significados dependendo do período histórico e do contexto sócio, político, econômico e cultural no qual está inserido'. Além disso, exerce papel fundamental na construção da identidade e define as formas de inserção do indivíduo no mundo. São as relações cotidianas que permitem essa construção tanto individual quanto social. Na vida adulta, o espaço do trabalho se constitui como mediador da construção e desenvolvimento dessa identidade ${ }^{2}$.

O trabalho traz satisfação ao homem, possibilitando reconhecimento social, promove a autoestima e possibilita a afirmação da identidade pessoal. Ele também determina a estrutura temporal do funcionamento da sociedade ${ }^{3}$.

Historicamente, pessoas com algum tipo de deficiência passaram por processos de segregação, marginalização e estigmatização. O movimento e lutas pelos direitos das pessoas com deficiência, em busca de igualdade iniciou a partir da década de 1960, relacionado à necessidade de acesso ao trabalho. $\mathrm{O}$ direito ao trabalho e a outras atividades pertinentes à vida do homem pode ser visto como resultado de movimentos sociais que resultaram em legislações, convenções, declarações, políticas e ações que contribuíram para a implementação de políticas sociais, das oportunidades e relações com as pessoas com deficiência ${ }^{4}$.

Dentre este aparato legal, no contexto brasileiro, está a Política Nacional para a Integração da Pessoa Portadora de Deficiência, em consonância com o Programa Nacional de Direitos Humanos, a Lei de Cotas, e atualmente a Convenção sobre os Direitos das Pessoas com Deficiência'.

$\mathrm{O}$ tema do acesso da pessoa com deficiência no mercado de trabalho vem sendo discutido em pesquisas que destacam o perfil da pessoa com deficiência ${ }^{6,7}$, as dificuldades e propostas para as barreiras da inclusão ${ }^{8,9}$, os debates a partir de experiências da inserção no trabalho $0^{9,10}$ e sobre a compreensão de como as pessoas com deficiência vivenciam a experiência do trabalho ${ }^{11}$.

As pessoas com deficiência apresentam dificuldades e podem precisar de ajudas para realizar atividades no dia a dia. Essas dificuldades podem estar relacionadas à mobilidade, à aprendizagem, à comunicação e prejudicam o desempenho das atividades realizadas em casa, na escola, no lazer ou no trabalho ${ }^{12}$. As ações direcionadas à infraestrutura dos espaços públicos, como a acessibilidade e os transportes adaptados são exemplos de respostas às necessidades das pessoas com deficiência para participar da vida social ${ }^{13}$.

Nessa direção, a tecnologia assistiva pode contribuir e apoiar o desempenho de atividades, incluindo o trabalho.
O termo tecnologia assistiva é definido como uma área de conhecimento interdisciplinar e que abrange produtos, recursos, metodologias, estratégias, práticas e serviços com a meta da promoção de funcionalidade, quer seja nas atividades, quer seja na participação, das pessoas com deficiência, que apresentam incapacidades ou redução na mobilidade ${ }^{14}$.

O presente estudo busca contribuir para a reflexão do tema trabalho e deficiência e tem por objetivos identificar aspectos que facilitam e/ou dificultam a inclusão de pessoas com deficiência no mercado trabalho e analisar a contribuição da tecnologia assistiva no trabalho na perspectiva das pessoas com deficiência física.

Trata-se da divulgação de parte dos resultados de uma pesquisa do Programa de Bolsas de Iniciação Científica da Universidade Federal de São Carlos, subvencionada pelo Conselho Nacional de Desenvolvimento Científico e Tencológico, durante os anos de 2013 e 2014.

\section{MÉTODO}

Trata-se de um estudo descritivo exploratório de natureza qualitativa, tipo de método que se preocupa com o universo de significados, motivos, aspirações, crenças, valores e atitudes inerentes aos atos, às relações $\mathrm{e}$ às estruturas sociais. A pesquisa qualitativa busca uma compreensão particular daquilo que estuda e não se preocupa com generalizações e sim com o aprofundamento e abrangência da compreensão do grupo estudado ${ }^{15}$.

\section{Caracterização dos participantes}

A pesquisa foi realizada na Unidade Saúde Escola (USE) da Universidade Federal de São Carlos (UFSCar) e também contou com indicações de sujeitos pela Organização Não Gorvernamental - Movimento de Informações Sobre Deficiências (Ong-Mid). A escolha de ambas deu-se pela viabilidade de selecionar pessoas com deficiência que atendessem aos critérios de inclusão, a saber: adultos com idades entre 18 e 60 anos, com deficiência física, que estavam ou não trabalhando e que tinham o trabalho como um papel importante e desejado para o futuro a partir de indicações dos terapeutas ocupacionais da USE e de consulta a prontuários.

A seleção foi feita a partir de um rastreamento que utilizou o instrumento "Lista de Identificação de Papéis Ocupacionais" ${ }^{\prime 16}$. Nesse instrumento, o sujeito aponta dez possíveis papéis, atribuindo um grau de importância para cada um e informa os papéis em relação ao desempenho nos tempos: passado, presente e futuro ${ }^{15}$. $\mathrm{O}$ instrumento 
foi utilizado para recrutar apenas os sujeitos que referissem desejo de buscar inserção no trabalho e que atribuíssem o mais alto grau de importância para esse papel. Com isso, os participantes foram 30 adultos com deficiências físicas variadas.

\section{Instrumento da coleta de dados}

Utilizou-se como instrumento de coleta de dados uma entrevista semiestruturada. A escolha deste tipo de método deu-se em função da entrevista favorecer a obtenção de informação através da fala individual e permite através de um porta-voz a representatividade de um grupo ${ }^{15}$.

Nesse sentido foi elaborado pelos pesquisadores um roteiro pensado para este fim. $\mathrm{O}$ instrumento foi dividido em dados de identificação: nome, idade, sexo, escolaridade, ocupação e as seguintes perguntas:

1) Você acha que é necessário algum tipo de ajuda para que você consiga trabalhar? Se sim, Quais?; 2) Você acha que equipamentos, adaptações, tecnologias podem ajudar para a realização do trabalho?; 3) Para se inserir no mercado de trabalho, quais qualidades você acha que uma pessoa deve ter? 4) O que é trabalhar, para você? 5) Como você avalia o mercado de trabalho para a pessoa com deficiência?

\section{Procedimentos éticos}

Como procedimentos éticos, a coleta de dados foi feita mediante a assinatura de um Termo de Consentimento Livre e Esclarecido - TCLE. A pesquisa foi aprovada pelo Comitê de Ética Institucional da Universidade Federal de São Carlos (UFSCar), sob o parecer de número 196.255, CAAE número 12838213.0.0000.5504. Após o aceite pelos sujeitos, foi apresentado aos usuários o Termo de Consentimento Livre e Esclarecido (TCLE), este assinado em duas vias, uma para o participante e outra para o pesquisador.

\section{Coleta e análise de dados}

Cada entrevista foi aplicada pessoalmente com cada sujeito de forma individual. As coletas foram realizadas na Unidade Saúde Escola (USE) e na Ong-Mid, em ambiente reservado. As entrevistas foram feitas por um único pesquisador e tiveram, em média, duração de 45 minutos. Todas as entrevistas foram gravadas e transcritas integralmente.

A análise de dados foi feita a partir da técnica de análise de conteúdo temática, que compreende três etapas: pré-análise, exploração do material, tratamento dos resultados obtidos e interpretação. Participaram das três etapas dois pesquisadores, os quais realizaram os procedimentos conjuntamente ${ }^{17}$.

A primeira etapa contou com a utilização dos procedimentos: leitura flutuante, hipóteses, objetivos e elaboração de indicadores que fundamentaram a interpretação. Na segunda etapa os dados foram codificados a partir das unidades de registro. Na última etapa foi elaborada a categorização, que consistiu na classificação dos elementos segundo as suas semelhanças e por diferenciação, com posterior reagrupamento, em função de características comuns, contradições e antagonismos de cada relato ${ }^{17}$.

A análise resultou em quatro categorias: 1. Representação social do trabalho; 2. Qualidades e requisitos para inserção no mercado de trabalho; 3. Tecnologia assistiva e trabalho e 4. Mercado de trabalho com problemas para pessoas com deficiências.

Como procedimento metodológico, destacaram-se as falas dos sujeitos em itálico e entre aspas ao longo do texto. Cada sujeito foi nominado pela letra "S", seguida do algarismo arábico respectivo, de acordo com a ordem de coleta das entrevistas.

\section{RESULTADOS E DISCUSSÃO}

Os dados de identificação dos 30 sujeitos $(n=30)$ e sua caracterização a partir de dados sociodemográficos como idade, gênero, diagnóstico e escolaridade revelaram um número superior em relação à quantidade de homens $(\mathrm{n}=19)$ do que de mulheres $(\mathrm{n}=11)$, com média de faixa etária de 38,2 anos, variando entre 22 e 59 anos. A Tabela 1 apresenta os dados dos 30 sujeitos.

A seguir são apresentadas as categorias de análise temática do conteúdo, obtidas a partir do discurso dos sujeitos.

\section{CATEGORIA 1- Representação social do trabalho}

Os entrevistados apontaram o trabalho como uma questão de sobrevivência vinculada à renda, à independência e à conquista de objetivos, além de ser entendido como realização pessoal e profissional e um importante papel social que traz um sentimento de pertencimento, de vitalidade e utilidade. Tais informações são ilustradas pelos relatos abaixo:

\footnotetext{
"É a realização pessoal. Além de um processo necessário para se sustentar como ser humano, o trabalho representa importante papel psicológico e social para o indivíduo"(S1);
} 
Cruz DMC, et al. O trabalho e a tecnologia assistiva na perspectiva de pessoas. Rev Ter Ocup Univ São Paulo. 2015 set.-dez:;26(3):382-9.

"É um prazer porque me sinto útil, ativo e feliz em ter o meu dinheiro para fazer o que eu quiser sem depender de ninguém, além conhecer novas pessoas e aprender a desenvolver os meus talentos dia após dia, isso é ótimo" (S8).

Tabela 1 - Caracterização dos sujeitos $(n=30)$

\begin{tabular}{|c|c|c|c|c|}
\hline Participantes & Idade & Gênero & Diagnóstico & Escolaridade \\
\hline S1 & 36 & M & Lesão Medular & Fundamental Completo \\
\hline S2 & 26 & M & Lesão de Plexo Braquial & Médio Incompleto \\
\hline S3 & 33 & $\mathrm{M}$ & Paralisia Cerebral & Superior Incompleto \\
\hline S4 & 50 & $\mathrm{M}$ & Paralisia Infantil & Superior Completo \\
\hline S5 & 45 & M & Deformidade Congênita & Médio Completo \\
\hline S6 & 50 & $\mathrm{~F}$ & Lesão Medular & Médio Incompleto \\
\hline S7 & 30 & $\mathrm{~F}$ & AVC & Superior Incompleto \\
\hline S8 & 22 & $\mathrm{~F}$ & Deformidade Congênita & Superior Incompleto \\
\hline S9 & 33 & $\mathrm{~F}$ & Paralisia Cerebral & Superior \\
\hline S10 & 31 & M & Amputação & Médio Completo \\
\hline S11 & 24 & M & Lesão Medular & Superior Completo \\
\hline S12 & 31 & $\mathrm{~F}$ & Lesão Medular & Superior Incompleto \\
\hline S13 & 33 & $\mathrm{~F}$ & Lesão Medular & Superior Completo \\
\hline S14 & 22 & $\mathrm{M}$ & Distrofia Muscular & Médio Completo \\
\hline S15 & 36 & $\mathrm{M}$ & Lesão Medular & Superior Completo \\
\hline S16 & 51 & $\mathrm{M}$ & Paralisia Infantil & Pós Graduado \\
\hline S17 & 51 & M & Lesão Medular & Superior Completo \\
\hline S18 & 34 & $\mathrm{~F}$ & Síndrome de Stickler & Fundamental Incompleto \\
\hline S19 & 41 & M & Lesão Medular & Fundamental Completo \\
\hline $\mathbf{S 2 0}$ & 49 & M & Lesão Medular & Médio Completo \\
\hline $\mathbf{S 2 1}$ & 49 & M & AVC & Superior Incompleto \\
\hline S22 & 53 & $\mathrm{~F}$ & Lesão Medular & Superior Completo \\
\hline $\mathbf{S 2 3}$ & 28 & $\mathrm{~F}$ & Lesão Medular & Médio Completo \\
\hline S24 & 53 & M & Paralisia Cerebral & Médio Completo \\
\hline $\mathbf{S 2 5}$ & 25 & M & Esclerose Múltipla & Superior Completo \\
\hline S26 & 58 & M & Lesão Medular & Fundamental Incompleto \\
\hline $\mathbf{S 2 7}$ & 42 & M & Lesão Medular & Médio Completo \\
\hline $\mathbf{S} 28$ & 42 & $\mathrm{~F}$ & Paralisia Cerebral & Médio Completo \\
\hline S29 & 34 & $\mathrm{M}$ & $\mathrm{AVC}$ & Médio Completo \\
\hline S30 & 33 & $\mathrm{~F}$ & Lesão de Plexo Braquial & Médio Incompleto \\
\hline
\end{tabular}

Assim, os entrevistados utilizaram as palavras "ser" e "ter", ou seja, trabalhar significa ter qualidade de vida, uma forma de ser mais independente, ter dinheiro, além de ser algo prazeroso e que precisa ser conquistado.
Desta forma, o trabalho assume a esfera central da sociedade enquanto processo que cria valor, elemento vital para a realização do ciclo produtivo do capital. A centralidade do trabalho é parte estruturante do processo 
de socialização humana, carregando consigo sentido e realizações a vida das pessoas. E na busca de uma vida cheia de sentido, a atividade laborativa transforma-se em elemento humanizador ${ }^{18}$.

Enquanto uma oportunidade de desenvolvimento pessoal e de aprendizagem foi referida a importância de fazer algo que se goste e que sinta prazer.

O prazer no trabalho está ligado à confiança, ao reconhecimento do valor profissional de cada membro do coletivo e as relações estabelecidas e que são geradoras de comportamentos motivacionais. Entretanto, a linha divisória entre o prazer e o sofrimento no trabalho é tênue e depende da relação estabelecida entre o homem e a organização do trabalho' ${ }^{2}$.

As entrevistas também mostraram que o estar trabalhando representava um reconhecimento pela sociedade e uma forma de sentirem-se produtivos.

A inclusão no trabalho pode trazer diversos benefícios, garantindo o aprendizado e uma nova visão de mundo para as pessoas com deficiência. Trabalhar pode permitir que essas pessoas possam ser reconhecidas como profissionais competentes e trazer um real sentimento de inclusão em sua comunidade, melhorar a autoestima e autonomia nas atividades diárias, constituindo-se assim parte integrante e participativa de uma sociedade produtiva ${ }^{6}$.

O trabalho também permite ao indivíduo a visão de si como agente de transformação social, possibilitando a inclusão da pessoa com deficiência na sociedade, uma vez que este é fundamental para garantir a cidadania e o sentimento de pertencimento à sociedade. Para a pessoa com deficiência, o sentimento de eficácia pelo trabalho e de sua produção, contribui também para a construção de uma identidade social e de reconhecimento de sua capacidade, fazendo com que esta se sinta parte da sociedade como um sujeito produtivo ${ }^{19}$.

Um dos participantes apontou a importância do espaço de trabalho para que pudesse demonstrar a sua competência, independente de possuir uma deficiência ou não:

\section{"Trabalhando e mostrando a "minha competência” fazia com que eu não me sentisse tão diferente" (S3).}

Assim, o sentir-se diferente ocorre em consequência de preconceitos estabelecidos na sociedade e que definem o indivíduo que possui alguma deficiência física como um ser diferente, imperfeito, estranho e incapaz. Além disso, a desinformação sobre a deficiência torna-se um dos principais problemas gerados pela sociedade, pois produz um desconhecimento sobre as reais incapacidades e limitações das pessoas com deficiência e também de suas potencialidades e possibilidades ${ }^{20}$.

\section{CATEGORIA 2-Qualidades e requisitos para inserção no mercado de trabalho}

De acordo com os entrevistados, a conquista de um emprego está vinculada à questão da qualificação (estudos, cursos, experiências em determinadas funções) como consta nos relatos a seguir.

"Em primeiro lugar ela deve ter qualificação para o trabalho, pois só assim ela será competente e o mercado exige competência" (S10).

"Pessoas com deficiência tem que estar sempre se qualificando, pois é um mercado competitivo” (S5).

"Acho que as cotas não têm sido de grande ajuda porque o que falta é a qualificação do deficiente. Não adianta ter cotas se não temos pessoas qualificadas para ocupar as vagas" (S20).

Além da qualificação formal e especifica, os entrevistados destacaram a necessidade de algumas características pessoais, tais como dedicação, caráter, responsabilidade, comprometimento, força de vontade e espírito de trabalho em equipe e colaboração com os colegas. Abaixo alguns relatos ilustrativos.

"Dedicação, esforço, facilidade no aprendizado, boa dicção, comprometimento com a empresa. (S10)"

"Cumprimento dos horários e tarefas, dinamismo, boa comunicação, objetividade, espirito de colaboração, ser gentil, simpático com todos a sua volta, caráter, honestidade e força de vontade para aprender sempre [...] (S11)"

"Hoje acima de tudo espirito de equipe e liderança (S1)"

A fala de um dos sujeitos refere à existência de barreiras que não são tão fáceis e rápidas de serem removidas, como as arquitetônicas, e que interferem na qualificação da pessoa com alguma deficiência:

"[...] as barreiras físicas são determinantes. Vivo numa cidade muito difícil em termos de facilidades de acesso, transporte, etc. Isso desanima o deficiente e ele acaba desistindo de estudar para ficar em casa a espera das soluções assistencialistas do Estado" (S26). 
A existência de barreiras seja de ordem arquitetônica, atitudinal ou a falta de recursos didáticos e inadequação dos métodos de ensino, dificultam o processo de escolarização e de preparo profissional específico de muitas pessoas com deficiência física. Com isso, embora haja a disponibilização de vagas para serem ocupadas por pessoas com alguma deficiência, ainda existem dificuldades na admissão dessa população, seja por falta de qualificação profissional quanto de preparo social, principalmente quando se exige o desempenho de habilidades mais complexas e específicas ${ }^{21}$.

\section{CATEGORIA 3- Tecnologia assistiva e trabalho}

Dentre os trinta entrevistados, vinte e oito participantes referiram que a tecnologia assistiva era necessária e importante para a pessoa com deficiência no ambiente de trabalho, como ilustrado abaixo por algumas falas.

"A tecnologia assistiva é muito importante porque nos permite mais conforto e isso resulta, certamente, em mais qualidade no trabalho" (S22).

"A tecnologia assistiva é necessária no trabalho porque eu não consigo nem ficar muito tempo sentado, nem muito tempo em pé, o dia inteiro tem que tá alternando porque dói muito minha coluna e pernas" (S.18).

Além disto, treze dos entrevistados apontaram a necessidade de algum tipo de ajuda técnica (tecnologia assistiva) para se chegar até o trabalho em virtude da falta de acessibilidade dos transportes públicos como uma barreira importante.

"Bom, o mercado para nós está ótimo, mas falta transporte para facilitar a nossa locomoção ao local de trabalho, pois as empresas dão o vale transporte, mas sabemos que ônibus, trem e metrô são precários em nosso país" (S.23).

"[...] há muito a ser melhorado para auxiliar a locomoção do individuo tanto no trajeto até o local de trabalho quanto na própria empresa" (S19).

Outro aspecto abordado foi a consequência da ausência de tecnologia assistiva no ambiente de trabalho para a pessoa com deficiência física, como consta no relato:

"Eu que trabalho quase o tempo todo utilizando computador e Internet, sinto falta do uso da tecnologia assistiva no dia a dia no meu trabalho, porque a instituição não se preocupa muito com isso e a gente acaba sofrendo por causa das dores e postura inadequada” (S.27).
Os relatos coletados nas entrevistas trazem questões importantes para a discussão. Por um lado têm-se problemas de acesso e acessibilidade aos transportes públicos. Por outro, as particularidades de cada pessoa com deficiência, o que exige que os postos de trabalho e as funções ocupadas por cada sujeito sejam cuidadosamente analisados.

Ao destacar a acessibilidade, os entrevistados associaram à tecnologia assistiva, ao transporte adaptado e aos aspectos relacionados à mobilidade. Entende-se a acessibilidade como a garantia de que todos tenham acesso a todas as áreas de seu convívio, como a espaços físicos, mobiliários, equipamentos urbanos, sistemas e meios de comunicação e informação ${ }^{15}$. Mas também transporte, serviços e instalações abertos ao público ou de uso público, tanto na zona urbana como na rural ${ }^{22}$.

A partir das respostas é possivel afirmar que a garantia à acessibilidade não está sendo efetivada para todos, impossibilitando a plena participação nos espaços sociais, especificamente o de trabalho.

No ambiente de trabalho, as barreiras arquitetônicas existem tanto dentro quanto fora deste, a exemplo da presença de escadas, paralelepípedos, prateleiras altas, ausência de rampas, elevadores e banheiros. Tais barreiras dificultam o acesso independente das pessoas com deficiência às empresas, comprometendo o direito de ir e vir livremente no trabalho ${ }^{21}$.

Destaca-se que a acessibilidade no contexto do trabalho pode estar atrelada à adequações/mudanças nos postos de trabalho com auxílio da ergonomia ou da tecnologia assistiva, ambos os aspectos encontrados nas falas dos entrevistados.

\section{CATEGORIA 4-Problemas no mercado de trabalho para pessoas com deficiências}

Os entrevistados relataram que há no mercado de trabalho alguns problemas que dificultam a sua inserção. As falas abaixo retratam estes problemas citados.

"[...] tem uma grande carência de qualificação profissional devido à dificuldade de acesso a escola ou cursos profissionalizantes [...]” (S2).

"[..] problemas de acessibilidade urbana, ônibus adaptados com elevador, estações de transporte acessivel etc. [...]" (S14).

"[...] ate mesmo dentro da empresa a qual vai trabalhar, por muitas empresas não terem uma estrutura física adequada, por serem obrigados a reservar as vagas $e$ por não terem acessibilidade dentro da empresa, acaba escolhendo pessoas que tenha uma deficiência mais leve 
$[\ldots] "(S 4)$.

"[...] Preconceito, pois muitos não acreditam que a pessoa possa desenvolver algum trabalho correto [...]” (S7).

Para todos os participantes a inclusão não se trata apenas da adaptação dos espaços públicos e privados, ou da aquisição de equipamentos de autoajuda. A inclusão no mercado de trabalho deve considerar também a sua permanência neste espaço.

Tais problemas apontados corroboram com depoimentos de diversos deficientes físicos que referem que as dificuldades de acesso, a escolarização e a capacitação específica, a ausência ou número restrito de transporte adaptado nas cidades e o preconceito constituem fatores que comprometem a entrada da pessoa com deficiência no mercado de trabalho ${ }^{23}$.

Nessa direção a Lei de Cotas não resolve os problemas que essa população enfrenta para acessar e permanecer no mercado de trabalho. Acredita-se que contratar a pessoa com deficiência apenas para cumprimento de Lei, sem remoção dos obstáculos existentes (desde barreiras arquitetônicas até atitudinais), continuará por perpetuar preconceitos existentes em relação a essas pessoas, dentre estes o de que elas não possuem competências para o mercado trabalho ${ }^{21}$.

$\mathrm{Na}$ atual sociedade, a eficácia constitui um fundamento primordial, ou seja, tudo é medido pela sua capacidade de produzir efeitos que atendam a uma normativa. Este princípio, por consequência, aniquila as singularidades individuais e impossibilita que aqueles que não correspondem a norma, por apresentarem necessidades peculiares, tenham acesso ao mercado de trabalho ${ }^{24}$. Movimentos de resistência a este modo de produção devem ser pensados a fim de que as pessoas com deficiência possam desempenhar em empresas e indústrias, ocupando postos

\section{REFERÊNCIAS}

1. Lobato BC. Pessoas com deficiência no mercado de trabalho: implicações da lei de cotas [Dissertação]. São Carlos: Universidade Federal de São Carlos, Centro de Educação e Ciências Humanas, Programa de Pós-Graduação em Educação Especial; 2009. Disponível em: http://www.bdtd.ufscar.br/htdocs/ tedeSimplificado//tde_busca/arquivo.php? $\operatorname{codArquivo=2258}$. de trabalho que valorizem o que de melhor elas podem oferecer em conjunto com os demais trabalhadores, pois de fato, em uma empresa inclusiva todos os funcionários trabalham juntos e possuem igualdade de oportunidades ${ }^{19,5}$.

\section{CONSIDERAÇÕES FINAIS}

As análises das entrevistas retrataram as principais dificuldades neste processo e apontaram a existência da fragilidade no cumprimento a Lei de Cotas. As barreiras presentes na contratação de pessoas com deficiência no mercado de trabalho estão para além da fiscalização, mas nos obstáculos sociais, educacionais e assistenciais, por isso a importância de ações afirmativas e do incentivo ao debate pelo acesso aos direitos dessas pessoas garantidos em Lei.

Reconhecem-se algumas limitações quanto a este tipo de estudo; por restringir-se a entrevistar apenas sujeitos com deficiência física. Acredita-se que pessoas com outros tipos de deficiência tais como a visual e auditiva pudessem trazer diferentes demandas em relação ao trabalho.

Ao pensar na potencialidade de métodos para favorecer a inclusão no trabalho, destaca-se a utilização da tecnologia assistiva e da ergonomia, que possibilitam adaptar o ambiente às pessoas, além da habilitação e reabilitação da pessoa com deficiência.

Há a necessidade de mais diálogos que permitam a reflexão sobre a adaptação obrigatória dos ambientes e dos postos de trabalho e de uma equipe interdisciplinar mínima de profissionais (tais como psicólogo, engenheiro de produção, terapeuta ocupacional, arquiteto, assistente social) que possam cuidar das questões relacionadas à inclusão dessas pessoas. Sugestões para futuras pesquisas devem envolver a investigação de casos bem sucedidos de inclusão no trabalho e verificar quais foram os métodos e as estratégias adotadas.
2. Lancman S. Psicodinâmica do trabalho. In: Cavalcanti A, Galvão C. Terapia ocupacional: fundamentação \& prática. Rio de Janeiro: Guanabara Koogan; 2007. p.271-7.

3. Gravina MER, Nogueira DP, Rocha LE. Reabilitação profissional em um banco: facilitadores e dificultadores no retorno ao trabalho. Rev Ter Ocup Univ São Paulo. 2003; 
14(3):19-26. DOI: http://dx.doi.org/10.11606/issn.22386149.v14i1p19-26.

4. Lancillotti SSP. Deficiência e trabalho: redimensionando o singular no contexto universal. Campinas, SP: Autores Associados; 2003. (Coleção polêmicas do nosso tempo, 85).

5. Marques AE, Storch JA. Empregabilidade e deficiência física: uma questão de responsabilidade social. In: Costa MPR. A pessoa com deficiência no mercado de trabalho. São Carlos: Pedro \& João Editores; 2012. p.79-106.

6. Rodrigues DS, Simonelli AP, Soares LBT, Camarotto JA. Caracterização das pessoas com deficiência em idade economicamente ativa e mapeamento das instituições de assistência atuantes no município de São Carlos. Cad Ter Ocup UFSCar. 2009;17(2):107-18. Disponível em: http:// www.cadernosdeterapiaocupacional.ufscar.br/index.php/ cadernos/article/view/101

7. Almeida MA, Veltrone AA. Perfil da pessoa com deficiência no mercado de trabalho na cidade de São Carlos - SP. Rev Educ Espec (Santa Maria). 2010;23(36):73-90. http://www. redalyc.org/articulo.oa?id=313127408006.

8. Simonelli AP. Contribuições da análise da atividade e do modelo social para a inclusão no trabalho de pessoas com deficiência [Tese]. São Carlos: Universidade Federal de São Carlos, Centro de Ciências Exatas e de Tecnologia, Programa de Pós-Graduação em Engenharia de Produção; 2009. Disponível em: http://www.bdtd. ufscar.br/htdocs/tedeSimplificado//tde_busca/arquivo. php? $\operatorname{cod}$ Arquivo $=2255$.

9. Carvalho-Freitas MN, Souto JF, Simas ALB, Costa NB, Santos LMM, Marques AL. Willingness to work with people with disabilities in future Brazilian professionals. Work. 2014;50(4);543-52. DOI: 10.3233/WOR-131812.

10. Assis AM, Carvalho-Freitas MN. Estudo de caso sobre a inserção de pessoas com deficiência numa organização de grande porte. REAd Rev Eletrônica Admin (Porto Alegre). 2014;20(2):496-528. Disponível em: http://www.seer.ufrgs. $\mathrm{br} / \mathrm{read} /$ article/view/42126.

11. Coelho CM, Sampaio RF, Mancini MC. Trabalhadores com deficiência: vivências de prazer e sofrimento. Psicol Soc. 2014;26(1):214-23. http://dx.doi.org/10.1590/S010271822014000100023 .

12. Bracciali LMP. Tecnologia assistiva: perspectiva de qualidade de vida para pessoas com deficiência. In: Vilarta R, Gutierrez GL, Carvalho THPF, Gonçalves A, organizadores. Qualidade de vida e novas tecnologias. Campinas: Ipes Editorial; 2007. p.105-19.
13. Simonelli AP, Camarotto JA. As políticas brasileiras e internacionais de incentivo ao trabalho de pessoas com deficiência: uma reflexão. Cad Ter Ocup UFSCar (São Carlos). 2011;19(3):333-42. DOI: 10.4322/cto.2011.006.

14. Cruz DMC, Emmel MLG. Associations among occupational roles, independence, assistive technology, and purchasing power of individuals with physical disabilities. Rev Latino-am Enfermagem. 2013;21(2):484-91. http://dx.doi. org/10.1590/S0104-11692013000200003.

15. Minayo MCS. O desafio do conhecimento: pesquisa qualitativa em saúde. 3a ed. São Paulo: Hucitec, Abrasco; 2007.

16. Cordeiro JR. Validade transcultural da lista de papéis ocupacionais para portadores de doença pulmonar obstrutiva crônica (DPOC) no Brasil [Dissertação]. São Paulo: Universidade Federal de São Paulo, Escola Paulista de Medicina, Programa de Pós-Graduação em Reabilitação; 2005. Disponível em: http://livros01.livrosgratis.com.br/ cp052560.pdf.

17. Bardin L. Análise de conteúdo. Lisboa: Edições 70; 1977.

18. Antunes R. Os sentidos do trabalho. São Paulo: Boitempo; 2000.

19. Santos AC, Neto UG, Rezende EO. Profissionalização da pessoa com deficiência: aspectos históricos. In: Costa MPR. A pessoa com deficiência no mercado de trabalho. São Carlos: Pedro \& João Editores; 2012. p.9-22.

20. Ferreira SL. Aprendendo sobre a deficiência mental: um programa para crianças. São Paulo: Memnon; 1998.

21. Tanaka EDO, Manzini EJ. O que os empregadores pensam sobre o trabalho da pessoa com deficiência? Rev Bras Educ Espec (Marília). 2005;11(2):273-94. http://dx.doi. org/10.1590/S1413-65382005000200008.

22. Correa PM. Acessibilidade: conceitos e formas de garantia. Rev Bras Educ Espec (Marília). 2009;15(1):171-2. http:// dx.doi.org/10.1590/S1413-65382009000100012

23. Instituto Brasileiro de Defesa dos Direitos da Pessoa Portadora de Deficiência (IBDD). Sem limite. Inclusão de portadores de deficiência no mercado de trabalho. Rio de Janeiro: SENAC Rio; 2002. p.37-96: Depoimentos.

24. Instituto Brasileiro de Defesa dos Direitos da Pessoa Portadora de Deficiência (IBDD). Sem limite. Inclusão de portadores de deficiência no mercado de trabalho. Rio de Janeiro: SENAC Rio; 2002. 